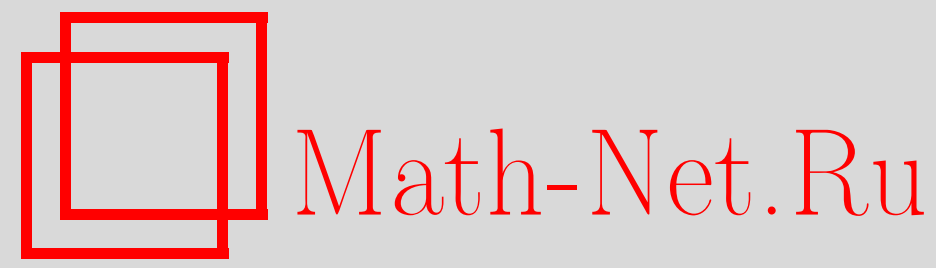

В. В. Конев, С. М. Пергаменщиков, Гарантированное оценивание параметра авторегрессии на основе обобщенного метода наименьших квадратов, Теория вероятн. и ее примен., 1996, том 41, выпуск 4, 765-784

DOI: https://doi.org/10.4213/tvp3201

Использование Общероссийского математического портала Math-Net.Ru подразумевает, что вы прочитали и согласны с пользовательским соглашением http://www.mathnet.ru/rus/agreement

Параметры загрузки:

IP : 54.172 .240 .79

26 апреля 2023 г., 17:11:35

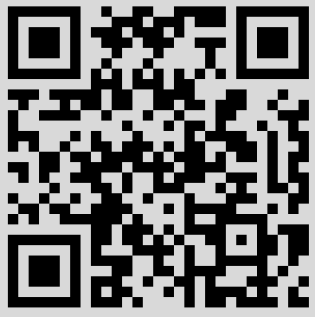




\title{
ГАРАНТИРОВАННОЕ ОЦЕНИВАНИЕ ПАРАМЕТРА АВТОРЕГРЕССИИ НА ОСНОВЕ ОБОБЩЕННОГО МЕТОДА НАИМЕНЬІІИХ КВАДРАТОВ
}

\begin{abstract}
Предлагается последовательная оценка параметра процесса авторегрессии первого порядка $(\mathrm{AP}(1))$, которая построена на основе обобщенного метода наименьших квадратов (ОМНK) (со специальным выбором весовых коэффициентов в сумме квадратов невязок). При некоторых естественных требованиях на распределение шума оценка является гарантированной в том смысле, что обеспечивает оценивание неизвестного параметра с любой заданной среднеквадратической точностью в момент прекрашения наблюдений. Предлагаемая оценка - в отличие от последовательной оценки МНК - обладает важным свойством равномерной асимптотической нормальности по параметру на всей прямой. С помощью этого результата показывается, что посл̀едовательная оценка ОМНК асимптотически оптимальна в минимаксном смысле при степенной функции потерь в широком классе последовательных и непоследовательных процедур.
\end{abstract}

Ключев ве слова и фразы: процесс авторегрессии, гарантированное оценивание, локальная асимптотическая нормальность, равномерная асимптотическая нормальность.

\section{$\S 1$. Введение}

Известно [1], [2], что в задачах оценивания параметров семейств распределений, обладающих свойством локальной асимптотической нормальности (ЛАН), последовательные оценки обычно не дают выигрыша (при степенных функциях потерь) по сравнению с оценками, построенными по выборке фиксированного объема. С другой стороны, известны примеры, показываюшие, что при нарушении свойства ЛАН для семейства распределений последовательные оценки могут иметь определенные преимущества. Один из таких примеров связан с моделью процесса авторегрессии, который играет фундаментальную роль в теории временных рядов [3].

*Томский университет, факультет прикладной математики и кибернетики, просп. Јенина, 36, 634050 Томск, Россия.

1) Работа выполнена при поддержке Российского фонда фундаментальных исследований, грант № 96-01-00189. 
Прощесс $\mathrm{AP}(1)\left\{x_{k}, k \geqslant 0\right\}$ определяется как решение разностного уравнения

$$
x_{k}=\lambda x_{k-1}+\varepsilon_{k}, \quad k \geqslant 1, \quad x_{0}=0,
$$

где $\lambda$ - постоянный параметр, $\left\{\varepsilon_{k}\right\}-$ ненаблюдаемая последовательность (шум) независимых одинаково распределенных (н.о.р.) случайных величин с $\mathbf{E} \varepsilon_{k}=0, \mathbf{E} \varepsilon_{k}^{2}=1$. В задачах оценивания параметра $\lambda$ по наблюдениям $x_{1}, \ldots, x_{n}$ наиболее часто используется оценка МНК

$$
\widehat{\lambda}_{n}=\frac{\sum_{k=1}^{n} x_{k-1} x_{k}}{\sum_{k=1}^{n} x_{k-1}^{2}}
$$

В [4] была предложена последовательная оценка МНК вида

$$
\lambda^{*}(H)=\frac{1}{H}\left[\sum_{k=1}^{N-1} x_{k-1} x_{k}+\alpha(H) x_{N-1} x_{N}\right],
$$

где $N=N(H)$ есть момент прекрашения наблюдений, определяемый равенством

$$
N(H)=\inf \left\{n \geqslant 1: \sum_{k=1}^{n} x_{k-1}^{2} \geqslant H\right\}, \quad \inf \{\varnothing\}=\infty,
$$

$H$ - параметр процедуры, $0<H<\infty ; \alpha(H)$ - поправочный множитель $(0<\alpha(H) \leqslant 1)$, который находится из уравнения

$$
\sum_{k=1}^{N-1} x_{k-1}^{2}+\alpha(H) x_{N-1}^{2}=H .
$$

Оценка (1.3) - в отличие от (1.2) - является несмещенной:

$$
\mathbf{E}_{\lambda} \lambda^{*}(H)=\lambda, \quad-\infty<\lambda<+\infty,
$$

и гарантированной в том смысле, что для всех $H>0$

$$
\sup _{-\infty<\lambda<\infty} \mathbf{E}_{\lambda}\left(\lambda^{*}(H)-\lambda\right)^{2} \leqslant \frac{1}{H}
$$

$\mathbf{E}_{\lambda}(\cdot)$ обозначает усреднение по распределению $\mathbf{P}_{\lambda}$ процесса $(1.1)$. В [5], [6] были построены гарантированные оценки параметров процесса авторегрессии произвольного порядка.

В [7] доказано, что последовательная оценка МНK (1.2), вычисленная в момент (1.4), обладает - в отличие от (1.2) - важным свойством равномерной по $\lambda \in[-1,1]$ асимптотической нормальности при $H \rightarrow \infty$, т.e.

$$
\lim _{H \rightarrow \infty} \sup _{\lambda \in[-1,1]} \sup _{x \in(-\infty, \infty)}\left|\mathbf{P}_{\lambda}\left\{\sqrt{H}\left(\hat{\lambda}_{N}-\lambda\right) \leqslant x\right\}-\Phi(x)\right|=0
$$


$\Phi(\cdot)$ - функщия распределения стандартного нормального закона с параметрами $(0,1)$.

В [8] развит метод, позволяющий в схеме гауссовского процесса (1.1) получить нижнюю границу для асимптотического риска:

$$
\varliminf_{H \rightarrow \infty} \sup _{|\lambda| \leqslant 1} \mathbf{E}_{\lambda} \omega\left(\sqrt{H}\left(\hat{\lambda}_{N}-\lambda\right)\right) \geqslant \mathbf{E} \omega(\xi),
$$

где $\omega(\cdot)$ - функщия потерь, удовлетворяющая обычным требованиям, $\widetilde{\lambda}_{N}$ - произвольная оценка по выборке $x_{1}, \ldots, x_{N} ; N=N(H)$ есть момент (1.4); $\xi$ - гауссовская случайная величина с параметрами $(0,1)$. Из (1.5), (1.6) непосредственно следует, что в случае невзрывной гауссовской авторегрессии (1.1) последовательная оценка (1.2), (1.4) оптимальна (для ограниченных функций потерь) в смысле минимума риска

$$
R(\widetilde{\lambda})=\varlimsup_{H \rightarrow \infty} \sup _{|\lambda| \leqslant 1} \mathrm{E}_{\lambda} \omega\left(\sqrt{H}\left(\widehat{\lambda}_{N}-\lambda\right)\right)
$$

T.e.

$$
\inf R(\tilde{\lambda})=R(\widehat{\lambda})
$$

где нижняя грань берется по всем последовательным процедурам $\widetilde{\lambda}=\left\{\left(N(H), \widetilde{\lambda}_{N}\right), H>0\right\} ; \widehat{\lambda}=\left\{\left(N(H), \widehat{\lambda}_{N}\right), H>0\right\}$.

В $[9$, глава $5, \S 4]$ была доказана равномерная асимптотическая нормальность последовательной оценки МНК (1.2), (1.4) для случая взрывного гауссовского процесса $(1.1)(|\lambda|>1)$. В [10] была установлена асимптотическая минимаксность гарантированной оценки параметра взрывного гауссовского процесса (1.1). Заметим, что условие гауссовости процесса (1.1) во взрывном случае является существенным для равномерной асимптотической нормальности последовательных оценок, построенных на основе МНК, поскольку их предельное распределение зависит от вида распределения шума. Последнее обстоятельство затрудняет использование этих оценок для решения практических задач, например, построения доверительных интервалов для неизвестного параметра при неизвестном распределении шума.

Цель данной работы - построить гарантированную оценку параметра $\lambda$, которая обладает свойством равномерной асимптотической нормальности по параметру на всей прямой для широкого класса распределений помех.

\section{§ 2, Основные результаты}

Оценка обобщенного метода наименьцих квадратов с последовательностью неотрицательных весовых коэффициентов $\left\{v_{k}\right\}_{k \geqslant 0}$ по наблюдениям $x_{1}, \ldots, x_{n}$ определяется равенством

$$
\lambda_{n}=\arg \min \sum_{k=1}^{n} v_{k-1}\left(x_{k}-\lambda x_{k-1}\right)^{2}=\frac{\sum_{k=1}^{n} v_{k-1} x_{k-1} x_{k}}{\sum_{k=1}^{n} v_{k-1} x_{k-1}^{2}} .
$$


Эта оценка совпадает с оценкой МНК при $v_{k} \equiv 1$. Определим последовательную оценку ОМНК формулами

$$
\begin{aligned}
\lambda^{*}(H) & =\frac{1}{\nu(H)}\left[\sum_{k=1}^{\tau-1} v_{k-1} x_{k-1} x_{k}+\beta(H) v_{\tau-1} x_{\tau-1} x_{\tau}\right] \\
\tau & =\tau(H)=\inf \left\{l \geqslant 1: \sum_{k=1}^{l} y_{k-1}^{2} \geqslant H\right\}, \quad 0<H<\infty \\
y_{k}^{2} & =v_{k}^{2} x_{k}^{2} \\
\nu(H) & =\sum_{k=1}^{\tau-1} v_{k-1} x_{k-1}^{2}+\beta(H) v_{\tau-1} x_{\tau-1}^{2} .
\end{aligned}
$$

Здесь $\beta(H)-$ поправочный множитель, который находится из условия отсутствия «перескока»у у последовательности сумм $\left\{\sum_{k=1}^{n} y_{k-1}^{2}, k \geqslant 1\right\}$ в момент $\tau(H)$ :

$$
\sum_{k=1}^{\tau-1} y_{k-1}^{2}+\beta(H) y_{\tau-1}^{2}=H .
$$

Заметим, что из (2.4) и (2.6) слецует, что для всех $H>0$

$$
0<\beta(H) \leqslant 1 \quad\left(\mathbf{P}_{\lambda} \text {-II.H. }\right)
$$

на множестве $\{\tau<\infty\}$.

На последовательность весовых коэффициентов $\left\{v_{k}\right\}$ наложим следуюшие требования:

1) $v_{k}$ измерима относительно $\sigma$-алгебры

$$
\mathcal{F}_{k}=\sigma\left\{x_{0}, \ldots, x_{k}\right\}, \quad k \geqslant 0
$$

2) $0 \leqslant v_{k} \leqslant 1, k \geqslant 0$

3) для всех $\lambda \in \Lambda \subseteq \mathbf{R}=(-\infty, \infty)$

$$
\sum_{k \geqslant 0} y_{k}^{2}=+\infty \quad\left(\mathbf{P}_{\lambda} \text {-п.н. }\right)
$$

4) для всех $k \geqslant 0$

$$
\lim _{a \rightarrow \infty} \sup _{\lambda \in \Lambda} \mathbf{P}_{\lambda}\left\{\left|y_{k}\right|>a\right\}=0
$$

5) (условие равномерной малости)

$$
\lim _{m \rightarrow \infty} \sup _{\lambda \in \Lambda} \mathbf{P}_{\lambda}\left\{\bigcup_{k \geqslant m}\left[y_{k}^{2}>\mu \sum_{j=1}^{k-1} y_{j}^{2}\right]\right\}=0
$$

цля всех $\mu>0$. 
На распределение шума $\varepsilon_{k}$ в (1.1), представляющего собой последовательность н.о.р. случайных величин, при изучении свойств оценки (2.2) будут налагаться условия:

A ) $\mathbf{E} \varepsilon_{1}=0, \mathbf{E} \varepsilon_{1}^{2}=1$

$\mathrm{A}_{2}$ ) распределение $\varepsilon_{k}$ имеет плотность $f(x)$, которая симметрична $(f(x)=f(-x))$ и монотонно не возрастает на $[0,+\infty)$;

$\mathrm{A}_{3}$ ) для некоторого $\delta>0$

$$
\mathbf{E} \varepsilon_{1}^{2+\delta}<\infty
$$

$\mathrm{A}_{4}$ ) плотность $f(x)$ распределения $\varepsilon_{k}$ кусочно-непрерывно дифференцируема, причем

$$
J(f)=\int_{-\infty}^{\infty} \frac{[\dot{f}(x)]^{2}}{f(x)} d x<\infty
$$

Свойства последовательной оценки (2.2)-(2.6) содержатся в следующих утверждениях

Теорема 1. Пусть для шума $\left\{\varepsilon_{k}\right\}$ выполнень условия $\left.\mathrm{A}_{1}\right)$, а последовательность $\left\{v_{k}\right\}$ удовлетворяет условиям 1)-5). Тогда оченка (2.2)-(2.6) является гарантированной, т.е. для любого $H>0$

$$
\sup _{\lambda \in \Lambda} \mathrm{E}_{\lambda}\left(\lambda^{*}(H)-\lambda\right)^{2} \leqslant \frac{1}{H}
$$

и равномерно асимптотически нормальной:

$$
\lim _{H \rightarrow \infty} \sup _{\lambda \in \Lambda} \sup _{x \in(-\infty, \infty)}\left|\mathbf{P}_{\lambda}\left\{\frac{\nu(H)}{\sqrt{H}}\left(\lambda^{*}(H)-\lambda\right) \leqslant x\right\}-\Phi(x)\right|=0 .
$$

3 а м е ч а н и е. При $\Lambda=[-1,1]$ и $v_{k} \equiv 1$ равенство (2.9) определяет равномерную асимптотическую нормальность гарантированной оценки (1.3) для невзрывного процесса (1.1). Этот результат легко получить непосредственно с помощью известной теоремы из [7].

Поскольку свойства последовательной оценки (2.2) зависят от выбора последовательности $\left\{v_{k}\right\}$, возникает естественный вопрос о том, можно ли в классе последовательностей, удовлетворяющих условиям 1)-5), выбрать такую, для которой оценка (2.2) равномерно асимптотически нормальна на всей прямой для достаточно широкого класса распределений помех.

Положим

$$
v_{k}= \begin{cases}1, & \left|x_{k}\right| \leqslant l_{k}, \\ \frac{\sqrt{1+l_{k}^{2}}}{\sqrt{1+x_{k}^{2}}}, & \left|x_{k}\right|>l_{k},\end{cases}
$$

где $\left\{l_{k}\right\}$ - такая последовательность неотрицательных чисел, что

$$
\lim _{k \rightarrow \infty} \frac{l_{k}}{\sqrt{k}}=0, \quad \lim _{k \rightarrow \infty} l_{k}=\infty
$$


Теорема 2. Пусть для последовательности $\left\{\varepsilon_{k}\right\}$ выполнены условия $\left.\left.\mathrm{A}_{1}\right), \mathrm{A}_{2}\right)$. Тогда последовательная оченка $(2.2)-(2.6)$ с последовательностью весовых коэффициентов (2.10) обладает свойствами гарантированности (2.8) и равномерной асимптотической нормальности (2.9) с $\Lambda=(-\infty, \infty)$. При этом для любого $H>0$ справедливо неравенство

$$
\sup _{-\infty<\lambda<\infty} \mathbf{E}_{\lambda} \tau(H) \leqslant \mathrm{E} \widehat{\tau}(H),
$$

гдe

$$
\begin{gathered}
\widehat{\tau}(H)=\inf \left\{k \geqslant 1: \sum_{j=1}^{k} V_{j-1}\left(\varepsilon_{j-1}\right) \geqslant H\right\}, \quad \varepsilon_{0}=x_{0}=0, \\
V_{k}(x)= \begin{cases}x^{2}, & |x| \leqslant l_{k}, \\
\frac{\left(1+l_{k}^{2}\right) x^{2}}{1+x^{2}}, & |x|>l_{k} .\end{cases}
\end{gathered}
$$

Далее покажем, что оценка (2.2)--(2.6) является оптимальной в некотором классе последовательных процедур. Для формулировки точного результата введем необходимые определения.

Предположим, что распределение шума $\varepsilon_{k}$ в (1.1) удовлетворяет требованиям $\left.\left.\mathrm{A}_{1}\right)-\mathrm{A}_{3}\right)$. Тогда совместная плотность $p\left(x_{1}, \ldots, x_{n} ; \lambda\right)$ распределения вектора $\left(x_{1}, \ldots, x_{n}\right)$ значений процесса $(1.1)$ при $|\lambda|<1$ обладает свойством локальной асимптотической нормальности (JАН) [11], т.е. имеет место равенство

$$
\begin{aligned}
\log & \frac{p\left(x_{1}, \ldots, x_{n} ; \lambda_{0}+u \varphi^{-1}\left(n, \lambda_{0}\right)\right)}{p\left(x_{1}, \ldots, x_{n} ; \lambda_{0}\right)} \\
& =u \Delta\left(n, \lambda_{0}\right)-\frac{u^{2}}{2}+\rho\left(n ; \lambda_{0}, u\right),
\end{aligned}
$$

где $\varphi(n, \lambda)=[n J(f)]^{1 / 2} / \sqrt{1-\lambda^{2}} ;$ случайная величина $\Delta\left(n, \lambda_{0}\right)$ асимптотически (при $n \rightarrow+\infty$ ) нормальна с параметрами $(0,1)$ по распределению $\mathbf{P}_{\lambda_{0}}$ процесса $(1.1)$, а случайная величина $\rho\left(n ; \lambda_{0}, u\right)$ стремится к нулю по вероятности $\mathbf{P}_{\lambda_{0}}$ при $n \rightarrow \infty$.

Пусть $\left\{\mathcal{F}_{k}\right\}_{k \geqslant 0}$ - последовательность $\sigma$-алгебр, порожденная процессом (1.1): $\mathcal{F}_{k}=\sigma\left(x_{0}, \ldots, x_{n}\right)$. Обозначим через $\mathfrak{M}$ класс последовательных и непоследовательных процедур $\widetilde{\lambda}=\left\{\left(t_{n}, \widetilde{\lambda}_{n}\right): n \geqslant 1\right\}$, где $t_{n}-$ такой марковский момент относительно последовательности $\left\{\mathcal{F}_{k}\right\}$, что

$$
\mathbf{E}_{\lambda} t_{n} \leqslant n
$$

для всех $n \geqslant 1, \tilde{\lambda}_{n}$ есть $\mathcal{F}_{t_{n}}$-измеримая случайная величина (оценка для $\lambda$ ). Для каждой процедуры $\tilde{\lambda} \in \mathfrak{M}$ определим риск по формуле

$$
R(\tilde{\lambda})=\sup _{f \in \mathcal{P}} \varlimsup_{n \rightarrow \infty} \sup _{\lambda \in \Lambda} \mathbf{E}_{\lambda} \omega\left(\psi_{n}\left(\tilde{\lambda}_{n}-\lambda\right)\right),
$$


где $\omega(x)=|x|^{\theta}, 0<\theta<2+\delta$ (здесь $\delta$ взято из условия $\mathrm{A}_{3}$ ) на распределение $\left.\varepsilon_{k}\right) ;\left\{\psi_{n}\right\}$ - нормировочная последовательность случайных величин, связанная с нормировочной функцией (2.14) предельным соотношением

$$
\lim _{\gamma \rightarrow 0} \varlimsup_{n \rightarrow \infty} \sup _{\left|\lambda-\lambda_{0}\right|<\gamma} \mathbf{P}_{\lambda}\left\{\left|\frac{\psi_{n} \sqrt{J(f)}}{\varphi\left(n, \lambda_{0}\right)}-1\right|>\mu\right\}=0
$$

для любых $\mu>0$ и $\lambda_{0} \in(-1,1) ; \Lambda$ - произвольное подмножество числовой прямой, содержащее некоторую окрестность из интервала $(-1,1)$; $\mathcal{P}$ - класс плотностей распределений шума $\varepsilon_{k}$, удовлетворяющих условиям $\left.\left.\mathrm{A}_{1}\right)-\mathrm{A}_{4}\right)$. Заметим, что критерий типа (2.16) с квадратической функцией потерь $(\theta=2)$ впервые использовался в задаче оценивания параметров авторегрессии в работе [15]. В следуюшей теореме устанавливается нижняя гранипа для риска (2.16).

Теорема 3. Пусть нормировочная последовательность $\psi_{n} 6$ (2.16) удовлетворяет соотношению (2.17). Тогда для любой последовательной процедуры $\widetilde{\lambda} \in \mathfrak{M}$ выполнено неравенство

$$
R(\tilde{\lambda}) \geqslant \mathbf{E} \omega(\xi)
$$

где $\xi$ - гауссовская случайная величина с параметрали $(0,1)$.

При изучении свойства оптимальности последовательного плана (2.2)-(2.6) предположим, что числовая последовательность $\left\{l_{k}\right\}$ в определении весовых коэффициентов (2.10), помимо (2.11), удовлетворяет дополнительному условию

$$
\sum_{k \geqslant 1} \frac{1}{l_{k}^{2+\delta}}<\infty
$$

где $\delta$ - число из условия $\left.A_{3}\right)$. Далее зададим последовательность порогов $\left\{H_{n}, n \geqslant 1\right\}$ формулами

$$
\begin{aligned}
H_{n} & =\left(h_{n}-1\right) \vee 0, \quad a \vee b=\max (a, b), \\
h_{n} & =\inf \left\{h>0: \mathbf{E}_{\lambda} \tau(h) \geqslant n\right\} .
\end{aligned}
$$

Этой последовательности порогов в соответствии с (2.2)-(2.6) отвечает последовательная процедура

$$
\lambda^{*}=\left\{\left(\tau\left(H_{n}\right), \lambda^{*}\left(H_{n}\right)\right), n \geqslant 1\right\}
$$

(полагаем $\tau(0)=\lambda^{*}(0)=0$ ). Благодаря выбору порогов $\left\{H_{n}\right\}$ последовательность моментов $\left\{\tau\left(H_{n}\right)\right\}$ удовлетворяет неравенству (2.15), а поэтому $\lambda^{*} \in \mathfrak{M}$.

Определим нормировочную последовательность формулой

$$
\psi_{n}=\frac{\nu\left(H_{n} \vee 1\right)}{\sqrt{H_{n} \vee 1}}
$$

где $\nu$ задается равенством (2.5). 
Теорема 4. Последовательная прочедура (2.21) является оптимальной в классе $\mathfrak{M}$ в смысле минимума риска (2.16) с нормировочной последовательностью (2.22), т.е.

$$
\min _{\tilde{\lambda} \in \mathfrak{M}} R(\tilde{\lambda})=R\left(\lambda^{*}\right)
$$

\section{§3. Вспомогательные утверждения}

Лемма 1 [7]. Пусть заданы: 1) семейство распределений $\left\{\mathrm{P}_{\lambda}(\cdot)\right.$, $\lambda \in \Lambda\}, \Lambda \subseteq \mathbf{R}=(-\infty, \infty)$, на борелевском пространстве $\left(\mathbf{R}^{\infty}, \mathcal{B}^{\infty}\right)$; 2) последовательность $\left\{\varepsilon_{k}\right\}$ случайных величин, независимых и одинаково распределенных относительно распределения $\mathbf{P}_{\lambda} c \mathbf{E}_{\lambda} \varepsilon_{n}=0 u$ $\mathbf{E}_{\lambda} \varepsilon_{n}^{2}=1$ при каждом $\lambda \in \Lambda$; 3) последовательность случайных величин $\left\{y_{k}\right\}_{k \geqslant 0}$, согласованная с $\left\{\mathcal{F}_{k}\right\}_{k \geqslant 0}$, аде $\mathcal{F}_{k}=\sigma\left(\varepsilon_{0}, \ldots, \varepsilon_{k}\right), \varepsilon_{0}=0, u$ удовлетворяющая условиям 3)-5) § 2;4) система марковских моментов $\{\tau(H), H>0\}$, определяемая равенством

$$
\tau(H)=\inf \left\{l \geqslant 1: \sum_{k=1}^{l} y_{k-1}^{2} \geqslant H\right\} .
$$

Tогдa

$$
\lim _{H \rightarrow \infty} \sup _{\lambda \in \Lambda} \sup _{x \in(-\infty, \infty)}\left|\mathbf{P}_{\lambda}\left\{\frac{1}{\sqrt{H}} \sum_{k=1}^{\tau(H)} y_{k-1} \varepsilon_{k} \leqslant x\right\}-\Phi(x)\right|=0 .
$$

Лемма 2. Пусть в прочессе (1.1) для $\left\{\varepsilon_{k}\right\}$ выполнено условие $\mathrm{A}_{2}$ ). Тогда для любой последовательности функиий $\left\{\varphi_{j}(\cdot)\right\}$, симметричных и неубывающих на $[0, \infty)$, выполняется неравенство

$$
\sup _{\lambda \in(-\infty, \infty)} \mathbf{P}_{\lambda}\left\{\sum_{j=1}^{k} \varphi_{j}\left(x_{j}\right)<c\right\} \leqslant \mathbf{P}\left\{\sum_{j=1}^{k} \varphi_{j}\left(\varepsilon_{j}\right)<c\right\} .
$$

ІІри любых $c \in(-\infty, \infty)$ u $k \geqslant 1$, где $\mathbf{P}_{\lambda}(\cdot)$ - распределение прочесса $\boldsymbol{x}_{j}$, соответствующее параметру $\lambda$.

Доказательство леммы 2 основывается на известной лемме Андерсона [12] и содержится в [13].

Лемма 3. Пусть $\omega(\cdot)-$ функция на прямой $(-\infty, \infty)$, удовлетворяющая условиям:

1) $\omega(-x)=\omega(x)$

2) $\omega(x) \geqslant \omega(y) n p u \quad 0<y<x$

3) $g(\mu)=\int \omega(x / \mu) e^{-x^{2}} d x<\infty \partial \Omega s$ вcex $\mu>0 u \lim g(\mu)=0 n p u$ $\mu \rightarrow \infty$.

Тогда для любой последовательной прочедуры $\tilde{\lambda} \in \mathfrak{M}$ и любых $\lambda_{0} \in(-1,1), \mu>0$ u $\gamma>0$ maкux, что $\left(\lambda_{0}-\gamma, \lambda_{0}+\gamma\right) \subset(-1,1)$, вы- 
полняется неравенство

$$
\begin{aligned}
\varliminf_{b \rightarrow \infty} \underset{n \rightarrow \infty}{\lim _{n \rightarrow \infty}}\left(\sup _{\left|\lambda-\lambda_{0}\right|<\gamma} \mathbf{E}_{\lambda}\left\{\omega\left(\varphi\left(n, \lambda_{0}\right)\left(\tilde{\lambda}_{t_{n}}-\lambda\right)\right)\right\}\right. \\
-\frac{1}{2 b} \int_{-b}^{b} \mathbf{E}_{\lambda_{0}+u \varphi^{-1}\left(n, \lambda_{0}\right)}\left\{\int_{-\infty}^{\infty} \frac{e^{-x^{2} / 2}}{\sqrt{2 \pi}}\right. \\
\\
\left.\left.\times \omega\left(\frac{x}{\varphi\left(t_{n} ; \lambda_{0}\right) / \varphi\left(n, \lambda_{0}\right)+\mu}\right) d x\right\} d u\right) \geqslant 0 .
\end{aligned}
$$

Д ок а з а те ль с т в о ле м м ы 3. Поскольку для распределений $\mathbf{P}_{\lambda}$ процесса $(1.1)$ для любого $\lambda_{0} \in(-1,1)$ выполнено свойство ЛАН (2.14), то неравенство (3.2) вытекает из теоремы 1 из [2].

Лемма 4. Пусть для последовательности $\left\{\varepsilon_{k}\right\}$ в (1.1) выполняются условия $\left.\left.\mathrm{A}_{1}\right), \mathrm{A}_{3}\right)$. Тогда для любых $\mu>0, \lambda_{0} \in(-1,1) u \gamma>0$ maкux, что $\left(\lambda_{0}-\gamma, \lambda_{0}+\gamma\right) \subset(-1,1)$, справедливо предельное соотночение

$$
\lim _{H \rightarrow \infty} \sup _{\left|\lambda-\lambda_{0}\right|<\gamma} \mathbf{P}_{\lambda}\left\{\left|\frac{\nu(H)}{H}-1\right|>\mu\right\}=0,
$$

где $\nu(H)$ определяется в (2.5) с помоцью весовой последовательности (2.10), удовлетворяющей условию (2.19).

Д ок а з а т л в с т в о л е м м 4. По определению $\nu(H)$, учитывая (2.7), для любого $m \geqslant 1, \mu>0$ имеем включение

$$
\begin{aligned}
& \left\{\left|\frac{\nu(H)}{H}-1\right|>\mu\right\} \subset\left\{\frac{1}{H} \sum_{k=1}^{\tau}\left(v_{k-1}-v_{k-1}^{2}\right) x_{k-1}^{2}>\mu\right\} \\
& \quad \subset\left\{\frac{1}{H} \sum_{k=1}^{m} x_{k-1}^{2}>\mu, \tau(H) \leqslant m\right\} \bigcup\left\{\frac{1}{H} \sum_{k=1}^{m} x_{k-1}^{2}>\frac{\mu}{2}, \tau(H)>m\right\} \\
& \bigcup\left\{\frac{1}{H} \sum_{k=m+1}^{\tau(H)}\left(v_{k-1}-v_{k-1}^{2}\right) x_{k-1}^{2}>\frac{\mu}{2}, \tau(H)>m\right\} \\
& \quad\left\{\frac{1}{H} \sum_{k=1}^{m} x_{k-1}^{2}>\frac{\mu}{2}\right\} \bigcup \Omega_{m}^{c},
\end{aligned}
$$

где

$$
\Omega_{m}=\bigcap_{k \geqslant m}\left[\left|x_{k}\right| \leqslant l_{k}\right]
$$

Покажем, что

$$
\lim _{m \rightarrow \infty} \sup _{\left|\lambda-\lambda_{0}\right|<\gamma} \mathbf{P}_{\lambda}\left(\Omega_{m}^{c}\right)=0
$$


Из (3.5) следует, что

$$
\mathbf{P}_{\lambda}\left(\Omega_{m}^{c}\right) \leqslant \sum_{k \geqslant m} \mathbf{P}_{\lambda}\left\{\left|x_{k}\right|>l_{k}\right\} \leqslant \sum_{k \geqslant m} \sup _{\left|\lambda-\lambda_{0}\right| \leqslant \gamma} \frac{\mathbf{E}_{\lambda}\left|x_{k}\right|^{2+\delta}}{l_{k}^{2+\delta}} .
$$

Оценим $\mathbf{E}_{\lambda}\left|x_{k}\right|^{2+\delta}$. Используя решение

$$
x_{k}=\sum_{i=1}^{k} \lambda^{k-i} \varepsilon_{i}
$$

уравнения (1.1), с помощью неравенства Гёльдера получаем

$$
\begin{aligned}
\sup _{k \geqslant 1} \sup _{\left|\lambda-\lambda_{0}\right|<\gamma} \mathbf{E}_{\lambda}\left|x_{k}\right|^{2+\delta} & \leqslant \frac{\mathbf{E}\left|\varepsilon_{1}\right|^{2+\delta}}{\left(1-|\lambda|^{(2+\delta) /(2(1+\delta))}\right)^{1+\delta}}\left(1-|\lambda|^{(2+\delta) / 2}\right)^{-1} \\
& \leqslant \frac{\mathbf{E}\left|\varepsilon_{1}\right|^{2+\delta}}{\left(1-r^{(2+\delta) /(2(1+\delta))}\right)^{2+\delta}}
\end{aligned}
$$

где $r=\left|\lambda_{0}-\gamma\right| \vee\left|\lambda_{0}+\gamma\right|$. Из (3.7), (3.9) и (2.19) вытекает предельное соотношение (3.6). Далее заметим, что согласно (3.8)

$$
\sup _{k \geqslant 1} \sup _{\left|\lambda-\lambda_{0}\right|<\gamma} \mathbf{E}_{\lambda} x_{k}^{2} \leqslant \frac{1}{1-r^{2}}
$$

Из включения (3.4) с помощью неравенства Чебышева и (3.10) получаем

$$
\begin{aligned}
\mathbf{P}_{\lambda}\left\{\left|\frac{\nu(H)}{H}-1\right|>\mu\right\} & \leqslant \frac{2}{\mu H} \sum_{k=1}^{m} \mathbf{E}_{\lambda} x_{k-1}^{2}+\mathbf{P}_{\lambda}\left(\Omega_{m}^{c}\right) \\
& \leqslant \frac{2 m}{\mu H\left(1-r^{2}\right)}+\sup _{\left|\lambda-\lambda_{0}\right|<\gamma} \mathbf{P}_{\lambda}\left(\Omega_{m}^{c}\right),
\end{aligned}
$$

где $\lambda \in\left(\lambda_{0}-\gamma, \lambda_{0}+\gamma\right)$. Применяя операцию $\lim _{m \rightarrow \infty} \lim _{H \rightarrow \infty}$ к этому неравенству и учитывая предельное соотношение (3.6), приходим к (3.3). Лемма 4 доказана.

Лемма 5. Пусть для последовательности $\left\{\varepsilon_{k}\right\}$ в (1.1) выполнены условия $\left.\left.\mathrm{A}_{1}\right), \mathrm{A}_{3}\right)$. Тогда для любых $\mu>0, \lambda_{0} \in(-1,1) u \gamma>0$ maких, что $\left(\lambda_{0}-\gamma, \lambda_{0}+\gamma\right) \subset(-1,1)$, справедливо предельное соотночение

$$
\lim _{H \rightarrow \infty} \sup _{\left|\lambda-\lambda_{0}\right|<\gamma} \mathbf{P}_{\lambda}\left\{\left|\frac{\tau(H)}{H}-\left(1-\lambda^{2}\right)\right|>\mu\right\}=0,
$$

где $\tau(H)$ - марковский момент (2.3), определдемый с помощью весовой последовательности (2.10), удовлетворяющей условию (2.19).

Дока за те льс т в о ле м мы 5 . Положим

$$
t_{*}=t_{*}(H)=\left[\left(1-\lambda^{2}\right) H-\mu H\right], \quad t^{*}=t^{*}(H)=\left[\left(1-\lambda^{2}\right) H+\mu H\right],
$$


где $[a]$ - целая часть числа $a$. Тогда

$$
\begin{aligned}
\mathbf{P}_{\lambda}\left\{\left|\frac{\tau(H)}{H}-\left(1-\lambda^{2}\right)\right|>\mu\right\} \leqslant & \mathbf{P}_{\lambda}\left\{\tau(H)<t_{*}(H)\right\} \\
& +\mathbf{P}_{\lambda}\left\{\tau(H)>t^{*}(H)\right\} .
\end{aligned}
$$

Из определения $\tau(H)$ следует, что для любого $m \geqslant 1$ и достаточно больших $H$

$$
\begin{aligned}
& \mathbf{P}_{\lambda}\left\{\tau(H)<t_{*}\right\}=\mathbf{P}_{\lambda}\left\{\sum_{k=1}^{t_{*}} V_{k-1}\left(x_{k-1}\right) \geqslant H\right\} \\
& \quad \leqslant \mathbf{P}_{\lambda}\left\{\sum_{k=1}^{t_{*}} V_{k-1}\left(x_{k-1}\right) \geqslant H, \Omega_{m}\right\}+\mathbf{P}_{\lambda}\left(\Omega_{m}^{c}\right) \\
& \quad \leqslant \mathbf{P}_{\lambda}\left\{s_{m}+\sum_{k=m}^{t_{*}-1} x_{k}^{2} \geqslant H\right\}+\mathbf{P}_{\lambda}\left(\Omega_{m}^{c}\right) \\
& \quad \leqslant \mathbf{P}_{\lambda}\left\{s_{m} \geqslant H^{3 / 4}\right\}+\mathbf{P}_{\lambda}\left\{\sum_{k=m}^{t_{*}-1} x^{2} \geqslant H-H^{3 / 4}\right\}+\mathbf{P}_{\lambda}\left(\Omega_{m}^{c}\right)
\end{aligned}
$$

где

$$
s_{m}=\sum_{k=1}^{m} V_{k-1}\left(x_{k-1}\right)
$$

Используя (3.10), опеним первое слагаемое в правой части (3.13):

$$
\sup _{\left|\lambda-\lambda_{0}\right|<\gamma} \mathbf{P}_{\lambda}\left\{s_{m} \geqslant H^{3 / 4}\right\} \leqslant \frac{\mathbf{E}_{\lambda} s_{m}}{H^{3 / 4}}=\frac{m}{\left(1-r^{2}\right) H^{3 / 4}} .
$$

Далее с помощью (1.1) получаем неравенство

$$
\begin{aligned}
\sum_{k=m}^{t_{*}-1} x_{k}^{2} & =\frac{\lambda^{2}}{1-\lambda^{2}}\left(x_{m-1}^{2}-x_{t^{*}-1}^{2}\right)+\frac{2 \lambda}{1-\lambda^{2}} \sum_{k=m}^{t_{*}-1} x_{k-1} \varepsilon_{k}+\frac{1}{1-\lambda^{2}} \sum_{k=m}^{t_{*}-1} \varepsilon_{k}^{2} \\
\leqslant & \frac{x_{m-1}^{2}}{1-\lambda^{2}}+\frac{2}{1-\lambda^{2}}\left|\sum_{k=m}^{t_{*}-1} x_{k-1} \varepsilon_{k}\right| \\
& +\frac{1}{1-\lambda^{2}} \sum_{k=m}^{t_{*}-1} \eta_{k}+H-\frac{\mu H}{\left(1-\lambda^{2}\right)}, \quad \eta_{k}=\varepsilon_{k}^{2}-1 .
\end{aligned}
$$

Отсюда выводим оценку для второго слагаемого в правой части (3.13):

$$
\begin{aligned}
& \mathbf{P}_{\lambda}\left\{\sum_{k=m}^{t_{*}-1} x_{k}^{2} \geqslant H-H^{3 / 4}\right\} \leqslant \mathbf{P}_{\lambda}\left\{\left|\sum_{k=m}^{t_{*}-1} \eta_{k}\right|>\mu H-2 H^{3 / 4}\right\} \\
& \quad+\mathbf{P}_{\lambda}\left\{x_{m-1}^{2} \geqslant\left(1-\lambda^{2}\right) \frac{H^{3 / 4}}{2}\right\}
\end{aligned}
$$




$$
+\mathbf{P}_{\lambda}\left\{\left|\sum_{k=m}^{t_{*}-1} x_{k-1} \varepsilon_{k}\right| \geqslant \frac{\left(1-\lambda^{2}\right) H^{3 / 4}}{4}\right\} .
$$

Запишем $\eta_{k}$ в виде

$$
\eta_{k}=\eta_{k}^{(1)}+\eta_{k}^{(2)}
$$

г де

$$
\begin{aligned}
& \eta_{k}^{(1)}=\eta_{k} \chi_{\left\{\left|\eta_{k}\right| \leqslant N\right\}}-\mathbf{E} \eta_{1} \chi_{\left\{\left|\eta_{1}\right| \leqslant N\right\}} \\
& \eta_{k}^{(2)}=\eta_{k} \chi_{\left\{\left|\eta_{k}\right|>N\right\}}-\mathbf{E} \eta_{1} \chi_{\left\{\left|\eta_{1}\right|>N\right\}},
\end{aligned}
$$

$N$ - положительное число, $\chi_{A}$ - индикатор события $A$. Отсюда и из (3.15) с помошью неравенства Чебышева и (3.10) получаем, что при достаточно больших $H$ и $\left(\lambda_{0}-\gamma, \lambda_{0}+\gamma\right)$

$$
\begin{aligned}
\mathbf{P}_{\lambda}\{ & \sum_{k=m}^{t_{*}-1} x_{k}^{2} \geqslant H-H^{3 / 4} \mid \leqslant \mathbf{P}_{\lambda}\left\{\left|\sum_{k=m}^{t_{*}-1} \eta_{k}^{(1)}\right| \geqslant \frac{\mu H}{2}-H^{3 / 4}\right\} \\
& +\mathbf{P}_{\lambda}\left\{\sum_{k=m}^{t_{*}-1}\left|\eta_{k}^{(2)}\right| \geqslant \frac{\mu H}{2}-H^{3 / 4}\right\}+\frac{2 \mathbf{E}_{\lambda} x_{m-1}^{2}}{\left(1-\lambda^{2}\right) H^{3 / 4}} \\
& +\frac{16}{\left(1-\lambda^{2}\right)^{2} H^{3 / 2}} \mathbf{E}_{\lambda}\left(\sum_{k=m}^{t_{*}-1} x_{k-1} \varepsilon_{k}\right)^{2} \leqslant \frac{t_{*} \mathbf{E}\left[\eta_{1}^{(1)}\right]^{2}}{\left(\mu H / 2-H^{3 / 4}\right)^{2}} \\
& +\frac{t_{*} \mathbf{E}\left|\eta_{1}^{(2)}\right|}{\mu H / 2-H^{3 / 4}}+\frac{2}{\left(1-r^{2}\right)^{2} H^{3 / 4}}+\frac{16 t_{*}}{\left(1-r^{2}\right)^{2} H^{3 / 2}} \\
\leqslant & \frac{4 H}{\left(\mu H-2 H^{3 / 4}\right)^{2}} \mathbf{E} \eta_{1}^{2} \chi_{\left\{\left|\eta_{1}\right| \leqslant N\right\}}+\frac{2 H}{\mu H-H^{3 / 4}} \mathbf{E}\left|\eta_{1}\right| \chi_{\left\{\left|\eta_{1}\right|>N\right\}} \\
& +\frac{2}{\left(1-r^{2}\right)^{2} H^{3 / 4}}+16\left(1-r^{2}\right)^{-2} H^{-1 / 2}
\end{aligned}
$$

Поскольку $\mathrm{E}\left|\eta_{1}\right|<\infty$, применяя операцию $\lim _{N \rightarrow \infty} \lim _{H \rightarrow \infty}$ к последнему. неравенству, выводим, что

$$
\lim _{H \rightarrow \infty} \sup _{\left|\lambda-\lambda_{0}\right|<\gamma} \mathbf{P}_{\lambda}\left\{\sum_{k=m}^{t_{*}-1} x_{k-1}^{2} \geqslant H-H^{3 / 4}\right\}=0 .
$$

Используя оценку (3.14) в неравенстве (3.13) и переходя к пределу сначала по $H \rightarrow \infty$, затем по $m \rightarrow \infty$ и принимая во внимание предельные соотношения $(3.6)$ и $(3.16)$, заключаем, что

$$
\lim _{H \rightarrow \infty} \sup _{\left|\lambda-\lambda_{0}\right|<\gamma} \mathbf{P}_{\lambda}\left\{\tau(H)<t^{*}\right\}=0 .
$$

Аналогично устанавливается, что

$$
\lim _{H \rightarrow \infty} \sup _{\left|\lambda-\lambda_{0}\right|<\gamma} \mathbf{P}_{\lambda}\left\{\tau(H)>t^{*}\right\}=0
$$


Из (3.12), (3.17) и (3.18) следует (3.11). Лемма 5 доказана.

Лемма 6. Пусть для последовательности $\left\{\varepsilon_{k}\right\}$ в (1.1) выполнено условие $\mathrm{A}_{2}$ ). Тогда для любого $r>0$

$$
\varlimsup_{H \rightarrow \infty} \sup _{\lambda \in(-\infty, \infty)} \mathbf{E}_{\lambda}\left[\frac{\tau(H)}{H}\right]^{\tau}<\infty,
$$

где $\tau(H)$ - марковский момент, определяемый с помощью весовой последовательности $v_{k}$, удовлетворяющей условиям (2.10), (2.11).

Д о к а з а т е ль с т в о л е м м ы 6 . Достаточно рассмотреть случай $r \geqslant 1$. Из невырожденности $\varepsilon_{k}$ следует, что для некоторого $\mu>0$

$$
\varkappa=\mathbf{E} \varepsilon_{1}^{2} \chi_{\left\{\left|\varepsilon_{1}\right| \leqslant \mu\right\}}>0 .
$$

Положим $\widetilde{\varepsilon}_{k}=\varepsilon_{k} \chi_{\left\{\left|\varepsilon_{k}\right| \leqslant \mu\right\}}$. Далее воспользуемся равенством

$$
\mathbf{E}_{\lambda}[\tau(H)]^{r}=r \int_{0}^{\infty} t^{r-1} \mathbf{P}_{\lambda}\{\tau(H)>t\} d t
$$

Из определения $\tau(H)$ с помошью леммы 2 получаем, что

$$
\begin{gathered}
\mathbf{P}_{\lambda}\{\tau(H)>t\}=\mathbf{P}_{\lambda}\left\{\sum_{k=1}^{[t]} V_{k-1}\left(x_{k-1}\right)<H\right\} \leqslant \mathbf{P}\left\{\sum_{k=2}^{[t]} V_{k-1}\left(\varepsilon_{k-1}\right)<H\right\} \\
\leqslant \mathbf{P}\left\{\sum_{k=2}^{[t]} V_{k-1}\left(\widetilde{\varepsilon}_{k-1}\right)<H\right\}=\mathbf{P}\left\{\sum_{k=1}^{[t]-1} \eta_{k}>e(t)-H\right\}
\end{gathered}
$$

где

$$
\eta_{k}=\mathbf{E} V_{k}\left(\widetilde{\varepsilon}_{k}\right)-V_{k}\left(\widetilde{\varepsilon}_{k}\right), \quad e(t)=\sum_{k=1}^{[t]-1} \mathbf{E} V_{k}\left(\widetilde{\varepsilon}_{1}\right)
$$

Пусть

$$
k_{0}=\inf \left\{j \geqslant 2: l_{j} \geqslant \mu\right\}
$$

(заметим, что $k_{0}<\infty$ в силу условия (2.11)). Тогда при $t>k_{0}$

$$
e(t)=\sum_{j=1}^{k_{0}-1} \mathbf{E} V_{j}\left(\widetilde{\varepsilon}_{1}\right)+\left([t]-k_{0}\right) \mathbf{E} \widetilde{\varepsilon}_{1}^{2} \geqslant x t+\varkappa_{0},
$$

где $\varkappa_{0}=\sum_{j=1}^{k_{0}-1} \mathbf{E} V_{j}\left(\widetilde{\varepsilon}_{1}\right)-\left(k_{0}+1\right) \varkappa$. Из $(3.21),(3.22)$ при $t>L_{0}(H)=$ $\left(H-x_{0}\right) / \varkappa$ с помошью неравенств Чебышева и Буркхольдера [14] получаем оценку

$$
\begin{aligned}
\mathbf{P}_{\lambda}\{\tau(H)>t\} & \leqslant \mathbf{P}\left\{\left|\sum_{k=1}^{[t]-1} \eta_{k}\right|>\varkappa\left(t-L_{0}(H)\right)\right\} \\
& \leqslant \frac{\mathbf{E}\left(\sum_{k=1}^{[t]-1} \eta_{k}\right)^{2 r+2}}{x^{2 r+2}\left(t-L_{0}(H)\right)^{2 r+2}} \leqslant \frac{B_{2 r+2} \mathbf{E}\left(\sum_{k=1}^{[t]-1} \eta_{k}^{2}\right)^{r+1}}{\varkappa^{2 r+2}\left(t-L_{0}(H)\right)^{2 r+2}}
\end{aligned}
$$




$$
\leqslant \frac{B_{2 r+2} t^{r+1} \mathbf{E} \eta_{1}^{2 r+2}}{x^{2 r+2}\left(t-L_{0}(H)\right)^{2 r+2}}
$$

где

$$
B_{l}=\left[\frac{18 l^{3 / 2}}{(l-1)^{1 / 2}}\right]^{l} .
$$

Из (3.20), (3.23) следует, что

$$
\begin{aligned}
\mathbf{E}_{\lambda}[\tau(H)]^{r} \leqslant & r \int_{0}^{L(H)} t^{r-1} d t+r \int_{L(H)}^{\infty} t^{r-1} \mathbf{P}_{\lambda}\{\tau(H)>t\} d t \\
= & L^{r}(H)+\frac{r B_{2 r+2} \mathrm{E}_{1}^{2 r+2}}{x^{2 r+2}} \int_{h(H)}^{\infty} \frac{\left(t+L_{0}(H)\right)^{2 r}}{t^{2 r+2}} d t \\
\leqslant & L^{r}(H)+\frac{2^{2 r-1} r B_{2 r+2} \mathrm{E} \eta_{1}^{2 r+2}}{x^{2 r+2}} \\
& \times\left[\int_{h(H)}^{\infty} t^{-2} d t+\left(L_{0}(H)\right)^{2 r} \int_{h(H)}^{\infty} t^{-2 r-2} d t\right] \\
\leqslant & L^{r}(H)+\frac{2^{r-1} r B_{2 r+2} \mathrm{E} \eta_{1}^{2 r+2}}{x^{2 r+2}} \\
& \times\left(\frac{1}{h(H)}+\frac{\left[L_{0}(H)\right]^{2 r}}{(2 r+1)[h(H)]^{2 r+1}}\right) .
\end{aligned}
$$

Здесь $L(H)=L_{0}(H)+h(H), h(H)=H+k_{0}$. Из неравенства (3.25) следует (3.19). Лемма 6 доказана.

Лемма 7. В условиях леммы 5 справедливо предельное соотношеพนе

$$
\lim _{H \rightarrow \infty} \sup _{\left|\lambda-\lambda_{0}\right|<\gamma} \mathbf{E}_{\lambda}\left|\frac{\tau(H)}{H}-\left(1-\lambda^{2}\right)\right|^{r}=0
$$

$\partial \Omega$ в $в$ cex $r>0$.

Утверждение леммы 7 следует из лемм 5, 6.

\section{§4. Доказательство основных утверждений}

Д о к а за те ль с т в о те о ремы 1 . Из определения $\tau(H)$ в $(2.3)$, принимая во внимание (2.7) и учитывая, что $\nu(H) \geqslant H$, получаем, что

$$
\begin{aligned}
\mathbf{E}_{\lambda}\left(\lambda^{*}(H)-\lambda\right)^{2} & =\mathbf{E}_{\lambda} \frac{1}{\nu^{2}(H)}\left[\sum_{k=1}^{\tau-1} v_{k-1} x_{k-1} \varepsilon_{k}+\beta(H) v_{\tau-1} x_{\tau-1} \varepsilon_{\tau}\right]^{2} \\
& \leqslant \frac{1}{H^{2}} \mathbf{E}_{\lambda}\left[\sum_{k=1}^{\tau-1} v_{k-1}^{2} x_{k-1}^{2}+\beta^{2}(H) v_{\tau-1}^{2} x_{\tau-1}^{2}\right] \leqslant \frac{1}{H} .
\end{aligned}
$$

Свойство равномерной асимптотической нормальности (2.9) последовательной оценки (2.2)--(2.6) следует из леммы 1. Теорема 1 доказана. 
Д ок а 3 а т е ль с т в о т е о р е м ы 2. Проверим, что для последовательности весовых коэффициентов (2.10) выполнены условия 1)-5) теоремы 1. Условия 1), 2) очевидны, а условие 3) следует из того, что

$$
\varlimsup_{k \rightarrow \infty} y_{k}^{2}=\varlimsup_{k \rightarrow \infty} V_{k}\left(x_{k}\right)>0 \quad\left(\mathbf{P}_{\lambda \text {-II.н. }}\right)
$$

для всех $\lambda \in(-\infty, \infty)$. Условие 4) вытекает из того, что $y_{j}^{2}=V_{j}\left(x_{j}\right) \leqslant$ $1+l_{j}^{2}$ для всех $j \geqslant 0$. Проверим условие 5). Имеем:

$$
\begin{aligned}
& \mathbf{P}_{\lambda}\left\{\bigcup_{k \geqslant m}\left[y_{k}^{2}>\mu \sum_{j=1}^{k-1} y_{j}^{2}\right]\right\}=\mathbf{P}_{\lambda}\left\{\bigcup_{k \geqslant m}\left[V_{k}>\mu \sum_{j=1}^{k-1} V_{j}\left(x_{j}\right)\right]\right\} \\
& \leqslant \mathbf{P}_{\lambda}\left\{\bigcup_{k \geqslant m}\left[1+l_{k}^{2}>\mu \sum_{j=1}^{k-1} V_{j}\left(x_{j}\right)\right]\right\} \\
& \quad \leqslant \sum_{k \geqslant m} \mathbf{P}_{\lambda}\left\{1+l_{k}^{2}>\mu \sum_{j=1}^{k-1} V_{j}\left(x_{j}\right)\right\} .
\end{aligned}
$$

Отсюда с помощью леммы 2 при $\varphi_{j}(x)=V_{j}(x)$ выводим неравенство

$$
\begin{aligned}
& \mathbf{P}_{\lambda}\left\{\bigcup_{k \geqslant m}\left[y_{k}^{2}>\mu \sum_{j=1}^{k-1} y_{j}^{2}\right]\right\} \leqslant \sum_{k \geqslant m} \mathbf{P}\left\{\sum_{j=1}^{k-1} V_{j}\left(\varepsilon_{j}\right)<\frac{1+l_{k}^{2}}{\mu}\right\} \\
& \quad \leqslant \sum_{k \geqslant m} \mathbf{P}\left\{\sum_{j=1}^{k-1} V_{j}\left(\tilde{\varepsilon}_{j}\right)<\frac{1+l_{k}^{2}}{\mu}\right\} \\
& =\sum_{k \geqslant m} \mathbf{P}\left\{\sum_{j=1}^{k-1} \eta_{j}>\sum_{j=1}^{k-1} \mathbf{E} V_{j}\left(\widetilde{\varepsilon}_{1}\right)-\frac{1+l_{k}^{2}}{\mu}\right\},
\end{aligned}
$$

где $\widetilde{\varepsilon}_{j}=\varepsilon_{j} \chi_{\left\{\left|\varepsilon_{j}\right| \leqslant a\right\}}, 0<a<\infty, \eta_{j}=\mathbf{E} V_{j}\left(\widetilde{\varepsilon}_{1}\right)-V_{j}\left(\widetilde{\varepsilon}_{j}\right)$, причем $\mathbf{E} \widetilde{\varepsilon}_{j}^{2}>0$. Далее, используя неравенство Чебышева и условие (2.11), для достаточно больших $m$ получаем

$$
\mathbf{P}_{\lambda}\left\{\bigcup_{k \geqslant m}\left[y_{k}^{2}>\mu \sum_{j=1}^{k-1} y_{j}^{2}\right]\right\} \leqslant \frac{\sum_{k \geqslant m} \mathbf{E}\left(\sum_{j=1}^{k-1} \eta_{j}\right)^{4}}{\left(\sum_{j=1}^{k-1} \mathbf{E} V_{j}\left(\widetilde{\varepsilon}_{1}\right)-\left(1+l_{k}^{2}\right) / \mu\right)^{4}} \leqslant \sum_{k \geqslant m} \frac{C}{k^{2}},
$$

где $C$ - некоторая постоянная, $0<C<\infty$.

Таким образом, условие 5) выполняется. Поэтому согласно теореме 1 оценка (2.2)-(2.6) с последовательностью весовых коэффициентов (2.10) обладает свойствами (2.8), (2.9). Неравенство (2.12) следует из оценки

$$
\mathbf{E}_{\lambda} \tau(H)=1+\sum_{k \geqslant 1} \mathbf{P}_{\lambda}\left\{\sum_{j=1}^{k} V_{j-1}\left(x_{j-1}\right)<H\right\}
$$




$$
\leqslant 1+\sum_{k \geqslant 1} \mathbf{P}\left\{\sum_{j=1}^{k} V_{j-1}\left(\varepsilon_{j-1}\right)<H\right\}=\mathbf{E} \widehat{\tau}(H) .
$$

Теорема 2 доказана.

Доказ а те ль с т в о те о рем ы 3 . Согласно условию на множество $\Lambda$ в определении риска (2.16) существуют числа $\lambda_{0} \in \Lambda$ и $\gamma_{0}>0$, для которых интервал $\left(\lambda_{0}-\gamma_{0}, \lambda_{0}+\gamma_{0}\right)$ содержится в пересечении $\Lambda \cap(-1,1)$. Поэтому для любых $0<\gamma \leqslant \gamma_{0}$ справедливо неравенство

$$
\begin{gathered}
\sup _{\lambda \in \Lambda} \mathbf{E}_{\lambda} \omega\left(\psi_{n}\left(\widetilde{\lambda}_{t_{n}}-\lambda\right)\right) \\
\geqslant[J(f)]^{-\theta / 2} \sup _{\left|\lambda-\lambda_{0}\right|<\gamma} \mathbf{E}_{\lambda} \omega\left(\frac{\psi \sqrt{J(f)}}{\varphi\left(n, \lambda_{0}\right)}\right) \omega\left(\varphi\left(n, \lambda_{0}\right)\left(\widetilde{\lambda}_{t_{n}}-\lambda\right)\right) \\
\geqslant[J(f)]^{-\theta / 2}\left[\sup _{\left|\lambda-\lambda_{0}\right|<\gamma} \mathbf{E}_{\lambda} \omega_{a}\left(\varphi\left(n, \lambda_{0}\right)\left(\widetilde{\lambda}_{t_{n}}-\lambda\right)\right)\right. \\
\left.-a^{\theta} \mathbf{E}\left|1-\omega_{a}\left(\frac{\psi \sqrt{J(f)}}{\varphi\left(n, \lambda_{0}\right)}\right)\right|\right]
\end{gathered}
$$

где $a>1, \omega_{a}(x)=(|x| \wedge a)^{\theta}, a \wedge b=\min (a, b)$. Отсюда следует, что для любых $a>1,0<\gamma \leqslant \gamma_{0}, \mu>0$ и $b>0$ имеет место оценка

$$
\begin{aligned}
\sup _{\lambda \in \Lambda} & \mathrm{E}_{\lambda} \omega\left(\psi_{n}\left(\tilde{\lambda}_{t_{n}}-\lambda\right)\right) \geqslant[J(f)]^{-\theta / 2} \\
\times & {\left[I_{n}(a, b)+\Delta_{n}(a, b)-a^{\theta} \sup _{\left|\lambda-\lambda_{0}\right|<\gamma} \mathbf{E}_{\lambda}\left|1-\omega_{a}\left(\frac{\psi_{n} \sqrt{J(f)}}{\varphi\left(n, \lambda_{0}\right)}\right)\right|\right], }
\end{aligned}
$$

где

$$
\begin{aligned}
I_{n}(a, b)= & \frac{1}{2 b} \int_{-b}^{b} \mathbf{E}_{\lambda_{0}+u \varphi^{-1}\left(n, \lambda_{0}\right)}\left\{\int_{-\infty}^{\infty} \omega_{a}\right. \\
& \left.\times\left(\frac{x}{\varphi\left(t_{n}, \lambda_{0}\right) / \varphi\left(n, \lambda_{0}\right)+\mu}\right) \frac{e^{-x^{2} / 2} d x}{\sqrt{2 \pi}}\right\} d u, \\
\Delta_{n}(a, b)= & \sup _{\left|\lambda-\lambda_{0}\right|<\gamma} \mathbf{E}_{\lambda} \omega_{a}\left(\varphi\left(n, \lambda_{0}\right)\left(\tilde{\lambda}_{t_{n}}-\lambda\right)\right)-I_{n}(a, b) .
\end{aligned}
$$

Используя неравенство Йенсена и условие (2.15), получаем

$$
\begin{aligned}
I_{n}(a, b)= & \mathbf{E} \omega(\xi) \frac{1}{2 b} \int_{-b}^{b} \mathbf{E}_{\lambda_{0}+u \varphi^{-1}\left(n, \lambda_{0}\right)}\left(\sqrt{\frac{t_{n}}{n}}+\mu\right)^{-\theta} d u \\
& +\frac{1}{2 \sqrt{2 \pi} b} \int_{-b}^{b} \mathbf{E}_{\lambda_{0}+u \varphi^{-1}\left(n, \lambda_{0}\right)}\left\{\int _ { - \infty } ^ { \infty } \left[\omega_{a}\left(\frac{x}{\sqrt{t_{n} / n}+\mu}\right)\right.\right.
\end{aligned}
$$




$$
\begin{aligned}
& \left.\left.-\omega\left(\frac{x}{\sqrt{t_{n} / n}+\mu}\right)\right] e^{-x^{2} / 2} d x\right\} d u \\
\geqslant & \mathbf{E} \omega(\xi) \frac{1}{2 b} \int_{-b}^{b}\left[\frac{\mathbf{E}_{\lambda_{0}+u \varphi^{-1}\left(n, \lambda_{0}\right)} \sqrt{t_{n}}}{\sqrt{n}}+\mu\right]^{-\theta} d u \\
& -\frac{1}{2 \sqrt{2 \pi} b} \int_{-b}^{b} \mathbf{E}_{\lambda_{0}+u \varphi^{-1}\left(n, \lambda_{0}\right)}\left\{\left(\sqrt{\frac{t_{n}}{n}}+\mu\right)^{-\theta}\right. \\
& \left.\times \int_{-\infty}^{\infty}|x|^{\theta} \chi_{\left\{|x| /\left(\sqrt{t_{n} / n}+\mu\right)>a\right\}} e^{-x^{2} / 2} d x\right\} d u \\
\geqslant & \frac{\mathbf{E} \omega(\xi)}{(1+\mu)^{\theta}}-\frac{1}{\sqrt{2 \pi}} \frac{1}{\mu^{\theta}} \int_{-\infty}^{\infty}|x|^{\theta} \chi_{\{|x|>a \mu} e^{-x^{2} / 2} d x
\end{aligned}
$$

где $\xi$ - гауссовская случайная величина с параметрами $(0,1)$. Отсюда и из (4.1) следует, что для любых $a>0,0<\gamma \leqslant \gamma_{0}, \mu>0$ и $b>0$ справедливо неравенство

$$
\begin{aligned}
\sup _{\lambda \in \Lambda} \mathbf{E}_{\lambda} \omega\left(\psi_{n}\left(\tilde{\lambda}_{t_{n}}-\lambda\right)\right) & \\
\geqslant[J(f)]^{-\theta / 2} & {\left[\frac{\mathbf{E} \omega(\xi)}{(1+\mu)^{\theta}}-\mu^{-\theta} \mathbf{E}|\xi|^{\theta} \chi_{\{|\xi|>a \mu\}}+\Delta_{n}(a, b)\right.} \\
& \left.\quad-a^{\theta} \sup _{\left|\lambda-\lambda_{0}\right|<\gamma} \mathbf{E}_{\lambda}\left|1-\omega_{a}\left(\frac{\psi_{n} \sqrt{J(f)}}{\varphi\left(n, \lambda_{0}\right)}\right)\right|\right]
\end{aligned}
$$

Переходя в этом неравенстве к нижнему пределу по $n \rightarrow \infty, b \rightarrow \infty$ и $\gamma \rightarrow 0$ и принимая во внимание условие (2.17) и лемму 3 , получим

$$
\lim _{n \rightarrow \infty} \sup _{\lambda \in \Lambda} \mathbf{E}_{\lambda} \omega\left(\psi_{n}\left(\widetilde{\lambda}_{t_{n}}-\lambda\right)\right) \geqslant[J(f)]^{-\theta / 2} \mathbf{E} \omega(\xi) \text {. }
$$

Далее заметим, что, поскольку класс $\mathcal{P}$ содержит гауссовскую плотность с параметрами $(0,1)$, то $\inf \{J(f): f \in \mathcal{P}\}=1$. Поэтому из (4.2) и (2.16) следует (2.18). Теорема доказана.

Д ок азательст в о те о рем ы 4. В силу условия на $\Lambda$ в риске (2.16) существуют числа $\lambda_{0} \in \Lambda$ и $\gamma_{0}>0$, для которых $\left(\lambda_{0}-\gamma_{0}, \lambda_{0}+\right.$ $\left.\gamma_{0}\right) \subset \Lambda \cap(-1,1)$. Далее установим следующее предельное соотношение для $H_{n}$ в $(2.20)$ :

$$
\lim _{n \rightarrow \infty} \sup _{\left|\lambda-\lambda_{0}\right|<\gamma_{0}}\left|\frac{n}{H_{n}}-\left(1-\lambda^{2}\right)\right|=0
$$

Согласно (2.12) последовательность $h_{n}^{*}$, определяемая равенством

$$
h_{n}^{*}=\inf \{h \geqslant 0: \mathbf{E} \widehat{\tau}(h) \geqslant n\},
$$


удовлетворяет для всех $n \geqslant 1$ неравенству

$$
\inf _{-\infty<\lambda<\infty} h_{n} \geqslant h_{n}^{*},
$$

и, следовательно, для всех $n$, начиная с некоторого номера $n_{0}$ такого, что $h_{n_{0}}^{*}>2$, выполняется неравенство

$$
\inf _{-\infty<\lambda<\infty} H_{n}>H_{n}^{*}=h_{n}^{*}-1 .
$$

По определению $h_{n}$

$$
\frac{\mathrm{E}_{\lambda} \tau\left(h_{n}\right)}{h_{n}} \geqslant \frac{n}{h_{n}}, \quad \frac{\mathrm{E}_{\lambda} \tau\left(H_{n}\right)}{H_{n}}<\frac{n}{H_{n}} .
$$

Отсюда следует, что при $n \geqslant n_{0}$

$$
\Delta\left(H_{n}, \lambda\right)-\frac{n}{H_{n} h_{n}}<\frac{n}{H_{n}}-\left(1-\lambda^{2}\right) \leqslant \Delta\left(h_{n}, \lambda\right)
$$

где

$$
\Delta(H, \lambda)=\frac{\mathbf{E}_{\tau}(H)}{H}-\left(1-\lambda^{2}\right) .
$$

Из леммы 7 следует, что

$$
\lim _{H \rightarrow \infty} \sup _{\left|\lambda-\lambda_{0}\right|<\gamma_{0}}|\Delta(H, \lambda)|=0 .
$$

Поэтому при достаточно больших $n$ из (4.5), (4.6) получаем

$$
\frac{n}{h_{n}} \leqslant 1+\sup _{h \geqslant h_{n}^{*}} \sup _{\left|\lambda-\lambda_{0}\right|<\gamma_{0}}|\Delta(h, \lambda)| \leqslant 2
$$

и, следовательно,

$$
\sup _{\left|\lambda-\lambda_{0}\right|<\gamma_{0}}\left|\frac{n}{H_{n}}-\left(1-\lambda^{2}\right)\right| \leqslant \frac{2}{H_{n}^{*}}+\sup _{h \geqslant H_{n}^{*}} \sup _{\left|\lambda-\lambda_{0}\right|<\gamma_{0}}|\Delta(h, \lambda)| .
$$

Переходя к пределу в этом неравенстве и учитывая (4.6), получим (4.3). Покажем теперь, что нормировочная последовательность (2.22) удовлетворяет соотношению (2.17). При $n \geqslant n_{0}$

$$
\begin{aligned}
\left|\frac{\psi_{n} \sqrt{J(f)}}{\varphi\left(n, \lambda_{0}\right)}-1\right|= & \left|\frac{\nu\left(H_{n}\right)\left(1-\lambda_{0}^{2}\right)^{1 / 2}}{\sqrt{H_{n}} \sqrt{n}}-1\right| \leqslant\left|\left(\frac{\left(1-\lambda_{0}^{2}\right)^{1 / 2}}{1-\lambda^{2}}\right)-1\right| \\
& +\sqrt{\frac{H_{n}}{n}}\left|\frac{\nu\left(H_{n}\right)}{H_{n}}-1\right|+\left|\frac{H_{n}}{n}-\frac{1}{1-\lambda^{2}}\right|
\end{aligned}
$$

Отсюда с помощью леммы 4, неравенства (4.4) и предельного соотношения (4.3) получаем (2.17). В силу теоремы 3 для риска (2.16) с нормировочной функцией (2.22) справедлива нижняя граница (2.18). Докажем, что для процедуры (2.21) неравенство (2.18) обращается в равенство. 
Сначала установим равномерную интегрируемость семейства случайных величин

$$
\zeta_{\lambda}(H)=\omega\left(\psi(H)\left(\lambda^{*}(H)-\lambda\right)\right),
$$

$\lambda \in \mathbf{R}, H \geqslant 1$. Для этого достаточно, чтобы

$$
\sup _{H \geqslant 1} \sup _{\lambda \in \mathbf{R}} \mathrm{E}_{\lambda}\left[\zeta_{\lambda}(H)\right]^{(2+\delta) / \theta}<\infty
$$

поскольку $0<\theta<2+\delta$. Учитывая, что $\omega(x)=|x|^{\theta}$, из $(2.2)$ и (4.7) с помошью неравенства Буркхольдера получаем

$$
\begin{aligned}
\mathbf{E}_{\lambda}[\zeta(H)]^{(2+\delta) / \theta} & =H^{-(2+\delta) / 2} \mathbf{E}_{\lambda}\left|\sum_{k=1}^{\tau-1} v_{k-1} x_{k-1} \varepsilon_{k}+\beta(H) x_{\tau-1} v_{\tau-1} \varepsilon_{\tau}\right|^{2+\delta} \\
& \leqslant \frac{B(2+\delta)}{H^{(2+\delta) / 2}} \mathbf{E}_{\lambda}\left|\sum_{k=1}^{\tau-1} y_{k-1}^{2} \varepsilon_{k}^{2}+\beta^{2}(H) y_{\tau-1}^{2} \varepsilon_{\tau}^{2}\right|^{(2+\delta) / 2}
\end{aligned}
$$

где $y_{k}=v_{k} x_{k}$, а постоянная $B(2+\delta)$ определяется равенством (3.24). Поскольку $\beta^{2}(H) \leqslant \beta(H)$ в силу $(2.7)$, используя неравенство Гёльдера, приходим к оценке

$$
\begin{aligned}
\mathbf{E}_{\lambda}\left[\zeta_{\lambda}(H)\right]^{(2+\delta) / \theta} \leqslant & \frac{B(2+\delta)}{H^{1+\delta / 2}} \mathbf{E}_{\lambda}\left[\sum_{k=1}^{\tau-1} y_{k-1}^{2}+\beta(H) y_{\tau-1}^{2}\right]^{\delta / 2} \\
& \times\left.\left|\sum_{k=1}^{\tau-1} y_{k-1}^{2}\right| \varepsilon_{k}\right|^{2+\delta}+\beta(H) y_{\tau-1}^{2}\left|\varepsilon_{\tau}\right|^{2+\delta} \mid \\
= & \frac{B(2+\delta)}{H} \mathbf{E}_{\lambda}\left[\sum_{k=1}^{\tau-1} y_{k-1}^{2}\left|\varepsilon_{k}\right|^{2+\delta}+\beta(H) y_{\tau-1}^{2}\left|\varepsilon_{\tau}\right|^{2+\delta}\right] \\
= & B(2+\delta) \mathbf{E}\left|\varepsilon_{1}\right|^{2+\delta},
\end{aligned}
$$

из которой следует (4.8). Далее заметим, что в силу теоремы 2 и неравенства (4.4) для последовательной процедуры (2.21) выполняется свойство равномерной асимптотической нормальности (2.9). Поэтому

$$
\varlimsup_{n \rightarrow \infty} \sup _{\lambda \in \Lambda}\left|\mathbf{E}_{\lambda} \zeta_{\lambda}\left(H_{n}\right)-\mathbf{E} \omega(\xi)\right|=0,
$$

где $\xi$ - гауссовская случайная величина с параметрами $(0,1)$. Отсюда и из (2.16) следует, что

$$
R\left(\lambda^{*}\right)=\mathbf{E} \omega(\xi) .
$$

Принимая во внимание (2.18), приходим к (2.23). Теорема 4 доказана. 


\section{СПИСОК ЛИТЕРАТУРЫ}

1. Ибрагимов И. А., Хасьмияский Р. 3. О последовательном оценивании. - Теория вероятн. и ее примен., 1974, т. 19, в. 2, с. 245-256.

2. Ефроймович C. Ю. О последовательном ощенивании в условиях локальной асимптотической нормальности. - Теория вероятн. и ее примен., 1980 , т. 25 , в. 1 , c. $30-43$.

3. Аядерсон T. Статистический анализ временных рядов. М.: Мир, 1976.

4. Борисов $B$. З., Конев $B$. В. Последовательное оценивание параметров дискретных процессов. -- Автоматика и телемеханика, 1977, № 10, с. 58-64.

5. Конев В. В., Пергамеяииков С. М. Последовательные планы идентификащии динамических систем. - Автоматика и телемеханика, 1981, № 7, с. 84-92.

6. Konev V. V., Lai T. L. Estimators with prescribed precision in stochastic regression models. - Sequential Anal., 1995, v. 14, p. 179-192.

7. Lai T. L., Siegmund D. Fixed-accuracy estimation of an autoregressive parameter. Ann. Statist., 1983, v. 11, № 2, p. 471-485.

8. Greenwood $P$. E., Shiryaev $A$. N. Asymptotic minimaxity of a sequential estimation for the first order autoregressive model. -- Stochastic, 1992, v. 38, p. 49-65.

9. Спокойный $B$. Г., ШІиряев $A$. Н. Статистические эксперименты и решения (асимптотическая теория). (В печати.)

10. Konev V. V., Pergamenshchikov S. M. On optimality of the fixed-accuracy estimate of the parameter in an explosive autoregressive process of the first order. - Sequential Anal., 1993, v. 12, № 1, p. 25-78.

11. Ибрагимов И. А., Хасьминский Р. З. Асимптотическая теория оценивания. М.: Наука, 1979.

12. Anderson $T$. $W$. The integral of a symmetric unimodal function over a symmetric convex set and some probability inequalities. - Proc. Amer. Math. Soc., 1955, v. 6, № 2, p. $170-176$.

13. Борисов B. 3., Конев В. В. О среднем времени наблюдения при последовательном оценивании параметров рекуррентных процессов. - Автоматика и телемеханика, 1981, № 10, с. 90-97.

14. Ширяев А. Н. Вероятность. М.: Наука, 1989.

15. Поляк Б. $Т$., Цыпкия Я. 3. Оптимальные методы оценивания коэффициентов авторегрессии при неполной информации. - Изв. АН СССР, сер. техн. кибернет., 1983 , № 1 , c. $118-126$. 